\title{
Comparison of four techniques for estimating temporal change of seismic velocity with passive image interferometry*
}

\author{
Zhikun Liu Jinli Huang * and Jiaojiao Li \\ Institute of Earthquake Science, China Earthquake Administration, Beijing 100036, China
}

\begin{abstract}
Passive image interferometry (PII) is becoming a powerful tool for detecting the temporal variations in the Earth's structure, which applies coda wave interferometry to the waveforms from the cross-correlation of seismic ambient noise. There are four techniques for estimating temporal change of seismic velocity with PII: moving-window cross-correlation technique (MWCCT), moving-window cross-spectrum technique (MWCST), stretching technique (ST) and moving-window stretching technique (MWST). In this paper, we use the continuous seismic records from a typical station pair near the Wenchuan $M_{\mathrm{S}} 8.0$ earthquake fault zone and generate three sets of waveforms by stacking cross-correlation function of ambient noise with different numbers of days, and then apply four techniques to processing the three sets of waveforms and compare their results. Our results indicate that the techniques based on moving-window (MWCCT, MWCST and MWST) are superior in detecting the change of seismic velocity, and the MWCST can give a better estimate of velocity change than the other moving-window techniques due to measurement error. We also investigate the clock errors and their influences on measuring velocity change. We find that when the clock errors are not very large, they have limited impact on the estimate of the velocity change with the moving-window techniques.
\end{abstract}

Key words: passive image interferometry; seismic ambient noise; temporal variation; moving-window cross-spectrum technique; stretching technique

CLC number: P315.4 Document code: A

\section{Introduction}

Measuring the temporal variation in subsurface seismic velocity has been a long-sought goal of seismology. In the early 1970 s, premonitory changes in seismic wave velocity (or ratio of $\mathrm{P}$ to $\mathrm{S}$ wave velocity) were widely documented (e.g., Semenov, 1969; Aggarwal et al., 1973; Whitcomb et al., 1973). However, these results were not reliable because the locations and origin times of the earthquakes used in these studies were poorly constrained. Subsequently, the velocity changes were measured by means of similar waveforms from active sources (e.g., Reasenberg and Aki, 1974; Vidale and $\mathrm{Li}, 2003$; Wang et al., 2008) and repeating earthquakes (e.g., Poupinet et al., 1984; Schaff and Beroza,

\footnotetext{
* Received 11 May 2010; accepted in revised form 20 July 2010; published 10 October 2010.

^Corresponding author. e-mail: hjl@seis.ac.cn

(c) The Seismological Society of China and Springer-Verlag Berlin Heidelberg 2010
}

2004; Peng and Ben-Zion, 2006). In addition, techniques based on coda wave interferometry (CWI) which took advantage of the phase and amplitude information were brought to measure the small changes in the medium (Snieder et al., 2002; Snieder, 2006). However, it was difficult to perform long-term and continuous monitoring velocity changes by using active sources or repeating earthquakes for their irregular sampling in time or space. More recently, employing the similar waveforms from seismic ambient noise correlation to monitor the change of seismic velocity, termed the passive image interferometry (PII), is rapidly developing. The temporal variations of seismic velocity were detected at volcanoes (Sens-Schönfelder and Wegler, 2006; Brenguier et al., 2008a; Duputel et al., 2009) and in earthquake fault zones (Wegler and Sens-Schönfelder, 2007; Brenguier et al., 2008b; Wegler et al., 2009; Liu and Huang, 2010) with PII.

PII is a method applying CWI to the waveforms from ambient noise correlation, thus the techniques for 
estimating velocity change are similar to traditional CWI, including moving-window cross-correlation technique (MWCCT) and moving-window cross-spectrum technique (MWCST). With the development of PII, stretching technique (ST) and moving-window stretching technique (MWST) were introduced (Sens-Schönfelder and Wegler, 2006). Hadziioannou et al. (2009) compared the MWCST and ST using the ultrasonic coda wave in a laboratory experiment. However, the signals from seismic ambient noise correlation are more complex, which are usually stacked with long time records and may contain the clock errors in different time periods.

In this paper, we focus on the data processing techniques for estimating the velocity change with PII, taking the data recorded near the Wenchuan $M_{\mathrm{S}} 8.0$ earthquake fault zone for an example. We apply four techniques for estimating the velocity changes (MWCCT, MWCST, ST and MWST) to three sets of waveforms, which are stacks of cross-correlation function (CCF) of ambient noise with different numbers of days. Comparing the results from different techniques, we discuss the impact factors on estimating the velocity change and individual advantages/disadvantages of these techniques.

\section{Techniques for estimating seismic ve- locity change}

First define a reference waveform $f_{\text {ref }}(t)$ and current waveform $f_{\text {cur }}(t)$. Both $f_{\text {ref }}$ and $f_{\text {cur }}$ are stacks of CCFs of seismic ambient noise, whereas $f_{\text {cur }}$ is computed in different time periods and $f_{\text {ref }}$ is a long time stacks to increase its stability. The change of seismic velocity can be estimated by comparing the two waveforms. Supposing the change of relative seismic velocity $\delta v / v$ is spatially homogeneous, the travel time shift $\delta t$ between $f_{\text {cur }}$ and $f_{\text {ref }}$ is proportional to lapse time $t$ (Poupinet et al., 1984; Snieder et al., 2002; Sens-Schönfelder and Wegler, 2006),

$$
\frac{\delta v}{v}=-\frac{\delta t}{t} .
$$

In other words, $f_{\text {cur }}$ is a compressed or stretched version of $f_{\text {ref }}$ due to the change in velocity. Four techniques are used to measure the change in relative seismic velocity and brief descriptions of them are as follows.

\subsection{Moving-window cross-correlation technique}

The moving-window cross-correlation technique (MWCCT) is a popular technique using CWI (e.g., Snieder et al., 2002; Grêt et al., 2005; Wang et al., 2008).
With this technique, a series of overlapping time windows are defined in coda wave and the time shifts between $f_{\text {cur }}$ and $f_{\text {ref }}$ in these windows are extracted by means of CCF. For a time window with length $2 t_{\mathrm{w}}$ centered at lapse time $t_{i}$, when the CCF of the two waveforms reach its maximal value, i.e.

$$
R\left(t_{\mathrm{s}}\right)=\frac{\int_{t_{i}-t_{\mathrm{w}}}^{t_{i}+t_{\mathrm{w}}} f_{\text {cur }}\left(t^{\prime}+t_{\mathrm{s}}\right) f_{\text {ref }}\left(t^{\prime}\right) \mathrm{d} t^{\prime}}{\sqrt{\int_{t_{i}-t_{\mathrm{w}}+t_{\mathrm{w}}}^{t_{\mathrm{w}}} f_{\text {cur }}^{2}\left(t^{\prime}\right) \mathrm{d} t^{\prime} \int_{t_{i}-t_{\mathrm{w}}}^{t_{i}+t_{\mathrm{w}}} f_{\text {ref }}^{2}\left(t^{\prime}\right) \mathrm{d} t^{\prime}}},
$$

the time shift $t_{\mathrm{s}}$ is equal to $\delta t_{i}$ (Snieder et al., 2002). The $\delta t_{i}$ can be achieved sub-sample precision using an interpolation of CCF. After all $\delta t_{i}$ are measured in the windows centered in an interest range of lapse time, the relative time shift $\delta t / t$ is estimated by a linear regression passing through zero and then $\delta v / v$ can be obtained according to equation (1). The error of $\delta v / v$ estimate is calculated as

$$
E_{\delta v / v}=\sqrt{\frac{\sigma_{\delta t}}{\sum_{i=1}^{M} t_{i}^{2}}}
$$

where $\sigma_{\delta t}$ is the fitting residual

$$
\sigma_{\delta t}=\frac{\sum_{i=1}^{M}\left(\delta t_{i}+t_{i} \frac{\delta v}{v}\right)^{2}}{M} .
$$

\subsection{Moving-window cross-spectrum technique}

Poupinet et al. (1984) introduced moving-window cross-spectrum technique (MWCST) to measure the temporal changes of seismic velocity between repeating earthquakes. After that, the MWCST is widely used to monitor the change in velocity (e.g., Ratdomopurbo and Poupinet, 1995; Schaff and Beroza, 2004; Brenguier et al., 2008a, b). This technique also begins with measuring time shift $\delta t_{i}$ at various lapse time $t_{i}$, but it works in the frequency domain. For a given small time window with length $2 t_{\mathrm{w}}$, the phase spectrum $\phi(f)$ is obtained from a cross-spectrum between $f_{\text {cur }}$ and $f_{\text {ref }}$ and the error $E_{\phi}$ of phase shift is estimated by

$$
E_{\phi}=\frac{1}{2 B_{\mathrm{w}} \times 2 t_{\mathrm{w}}}\left(\frac{1}{c^{2}}-1\right)
$$

where $c$ is the coherency and $B_{\mathrm{w}}$ is the spectrum width. If the time shift $\delta t_{i}$ is a constant in the window, the phase spectrum $\phi(f)$ is linearly proportional to the frequency $f$ :

$$
\phi(f)=2 \pi \delta t_{i} f
$$


Therefore, $\delta t_{i}$ can be estimated by a regression that takes into account the phase error $E_{\phi}$ as a weighting factor. Finally, $\delta v / v$ and its error are calculated in the same way as in the MWCCT described above.

\subsection{Stretching technique (ST)}

Because uniform velocity change can result in a compressed or stretched version of waveforms, SensSchönfelder and Wegler (2006) proposed a new technique to directly estimate $\delta v / v$ by compressing or stretching waveform along time axis, known as stretching technique (ST). A stretched version of $f_{\text {cur }}$ is written as

$$
f_{\operatorname{cur}(\varepsilon)}(t)=f_{\text {cur }}[t(1+\varepsilon)],
$$

where $\varepsilon=-\delta t / t$ is the stretching coefficient. By adjusting $\varepsilon$, the maximum of cross-correlation between $f_{\operatorname{cur}(\varepsilon)}$ and $f_{\text {ref }}$ can be got by

$$
R(\varepsilon)=\frac{\int f_{\operatorname{cur}(\varepsilon)}(t) f_{\text {ref }}(t) \mathrm{d} t}{\sqrt{\int f_{\operatorname{cur}(\varepsilon)}^{2}(t) \mathrm{d} t \int f_{\text {ref }}^{2}(t) \mathrm{d} t}},
$$

where $\varepsilon$ is an estimate of $\delta v / v$ from equation (1).

\subsection{Moving-window stretching technique (MWST)}

The quality of measurements with single-window ST can be only evaluated using the maximum of cross-correlation. In order to investigate the stability and estimate error of ST, repeating this technique in a series of time windows is adopted by some researchers (Sens-Schönfelder and Wegler, 2006; Meier et al., 2010). We call this technique as moving-window stretching technique (MWST). The $\delta v / v$ and its measurement error are obtained by calculating the mean and variance of all local $\delta v / v$ estimates in the time windows.

\section{Data processing and results com- parison}

\subsection{Data processing}

In our previous work, we detected the temporal variations in seismic velocity around the $M_{\mathrm{S}} 8.0$ Wenchuan earthquake fault zone with MWCST (Liu and Huang, 2010). In this paper, we will re-examine the estimate of temporal velocity variations with four techniques, taking the station pair AXI-JJS as an example. The two stations with an inter-station distance of $71 \mathrm{~km}$ are about 110-120 km from the epicenter (Figure 1). The same broadband seismometer, CMG-3ESPC, is fixed at the two stations. We adopt vertical component records of continuous waveforms from March 2007 to March 2009.

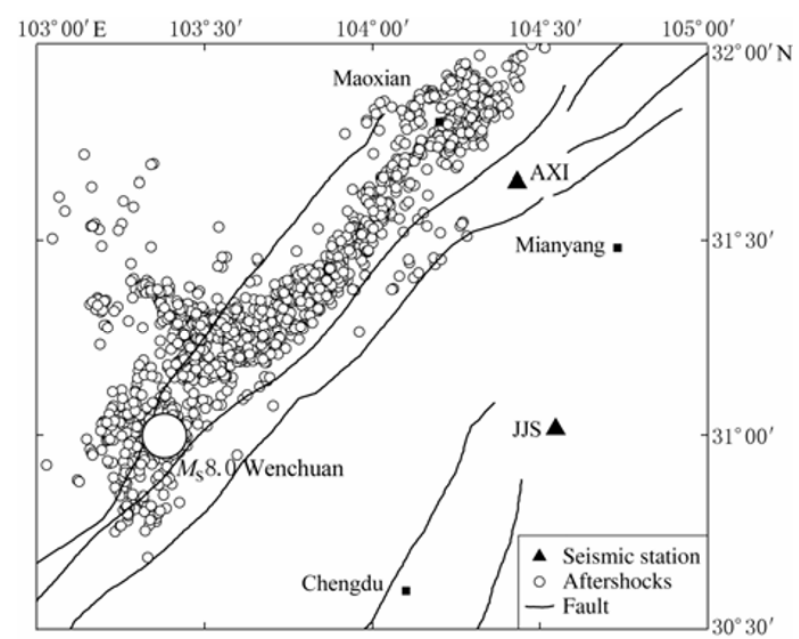

Figure 1 Distribution of seismic stations and earthquakes. The big and small circles denote the main shock of Wenchuan $M_{\mathrm{S}} 8.0$ earthquake and its aftershocks, respectively.

The ambient noise data processing follows the method described by Bensen et al. (2007). We clip the continuous data as daily segments and reject the segments which are shorter than 10 hours. After removing the mean and trend of data, we apply a temporal normalization by running-absolute-mean method with the window length of $15 \mathrm{~s}$ and spectral whitening between $0.08 \mathrm{~Hz}$ and $1.0 \mathrm{~Hz}$ to obtain single-station seismic ambient noise.

The empirical Green's function (EGF) between two stations can be extracted from the cross-correlation of seismic ambient noise (Shapiro and Campillo, 2004; Sabra et al., 2005). Repeating the process in different time periods provides similar waveforms to measure the change of seismic velocity (Sens-Schönfelder and Wegler, 2006). We stack all CCF with a length of 14 months before the main shock as the reference EGF $f_{\text {ref. In order to }}$ test the techniques in the presence of different data qualities, we compute daily EGFs $f_{\text {cur }}$ with three stack times, i.e., 11-day, 31-day and 61-day, respectively.

Figure 2 shows the daily EGF profile with 61-day stacks from March 2007 to March 2009. It is clear that the surface waves mainly with lapse time of 30-50 s are very stable in whole studied period. Although the stability of EGF decreases with increasing of the lapse times, the coda waves, even to $\pm 150 \mathrm{~s}$, still maintain relatively high continuity and stability. In the study of passive imaging, the signal-to-noise ratio (SNR) of direct wave, which is defined by the ratio of the maximal amplitude of surface wave to the mean-square-root of a trailing 
noise window, is used to characterize the ability of reconstructing Green's function from ambient noise (Bensen et al., 2007). We compute the SNR of daily EGF with three stack times and find that the SNR decreases with shortening stack times (Figure 3). The SNRs are fluctuant during the studied time period, but do not show obvious drop after the main shock. The similarity degree of the two waveforms can be quantified by their maximum of CCF, which also be used to study the medium changes with CWI (Snieder et al,
2002; Grêt et al, 2005). Figure 4 shows the correlation coefficients of current and reference EGFs in both coda wave windows (50-110 s) of positive and negative part of EGFs. We can see two remarkable decreases of correlation coefficient: one is in the time immediately after the main shock; the other is in the summer of 2007. Whether the decreases of correlation coefficient reflect the physical change in the medium or other signal will be discussed as below.
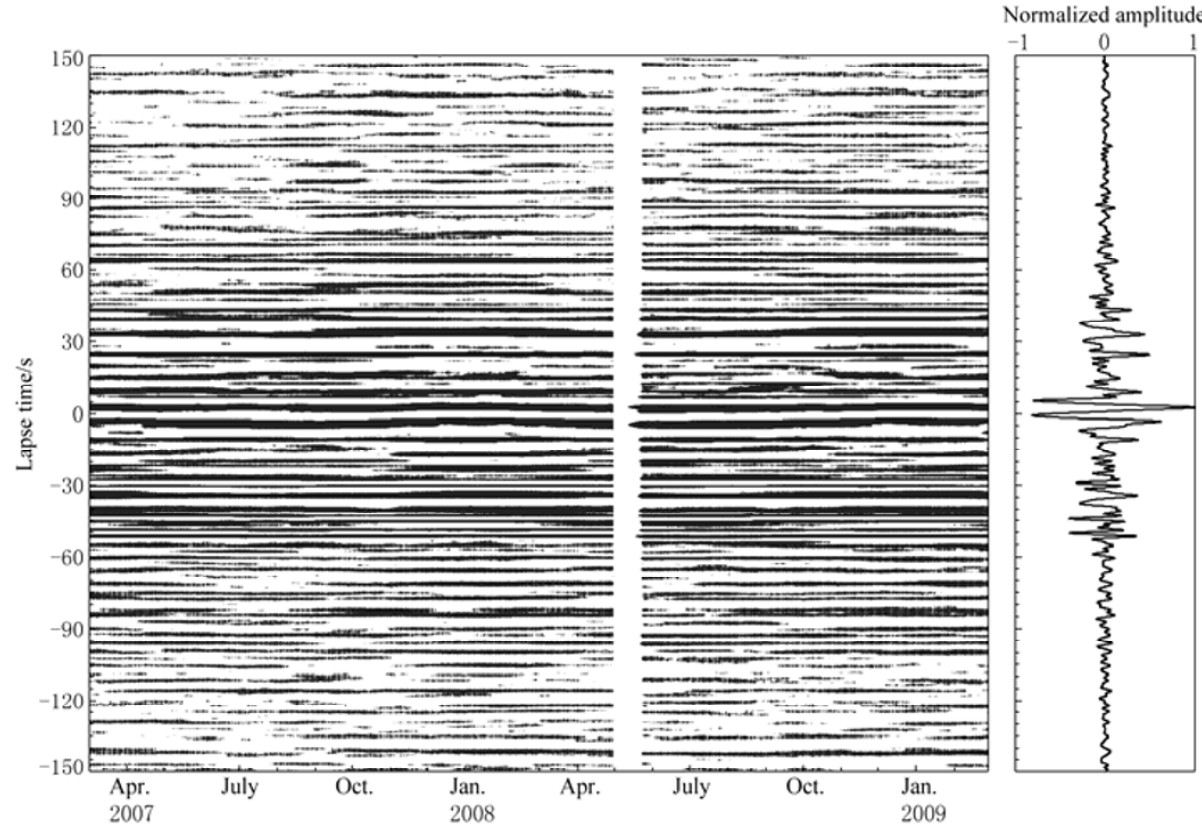

Figure 2 The current and reference empirical Green's functions (EGFs) of 61-day stacks from March 2007 to March 2009. The black wiggles correspond to positive amplitudes of current EGF. The waveform in the right box represents the reference EGF which is a stack of cross-correlation functions from March 1, 2007 to May 11, 2008.

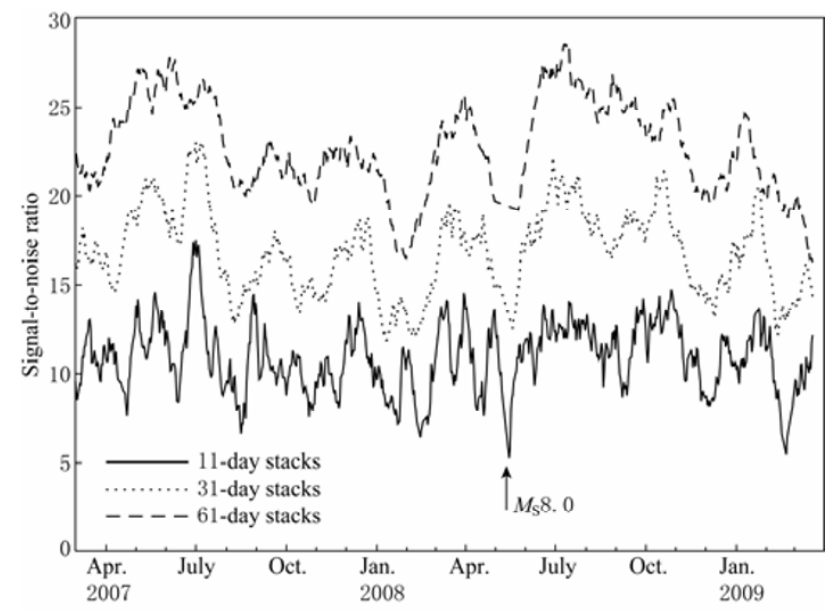

Figure 3 SNR of current EGF for 11-day, 31-day and 61-day stacks, respectively. The arrow denote the origin time of the Wenchuan $M_{\mathrm{S}} 8.0$ earthquake.

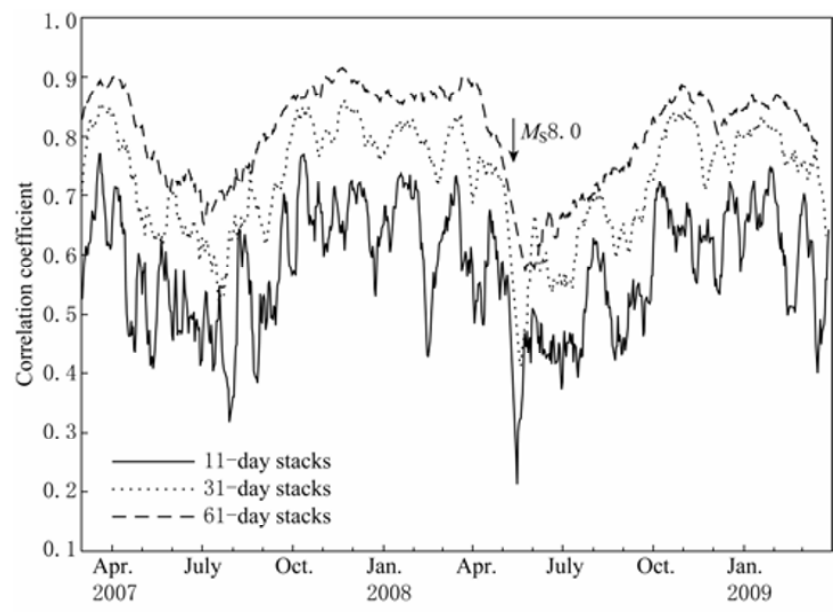

Figure 4 Temporal variations of correlation coefficient of current and reference EGFs for 11-day, 31-day and 61-day stacks, respectively. 


\subsection{Parameters design}

In order to obtain a comparable result by using three stack times, we design uniform computational parameters to estimate the seismic velocity change. The parameters are usually decided by the data quality, interstation distance, interest frequency band, and so on. In this study we choose parameters as follows.

The first parameter to be determined is the range of lapse time in which the current and reference EGFs are compared. Xu and Song (2009) selected the Rayleigh wave part of EGF and some other researchers paid more attentions to the coda wave which is more sensitive to the change of seismic velocity (e.g., Brenguier et al., 2008b; Wegler et al., 2009; Liu and Huang, 2010). Here we only use coda wave to estimate velocity change. The uniform range of lapse time should be determined based on the data of 11-day stacks, due to the lowest correlation with the shortest stack times (Figure 3). We compute the correlation coefficient in non-overlapping windows for the data of 11-day stacks (Figure 5). We can see that correlation coefficients decrease with the increase of lapse time, but basically maintain above 0.5 till the lapse time of $110 \mathrm{~s}$. So we choose the lapse time range of $50-110 \mathrm{~s}$ for both the positive and negative parts of EGF to estimate velocity change.

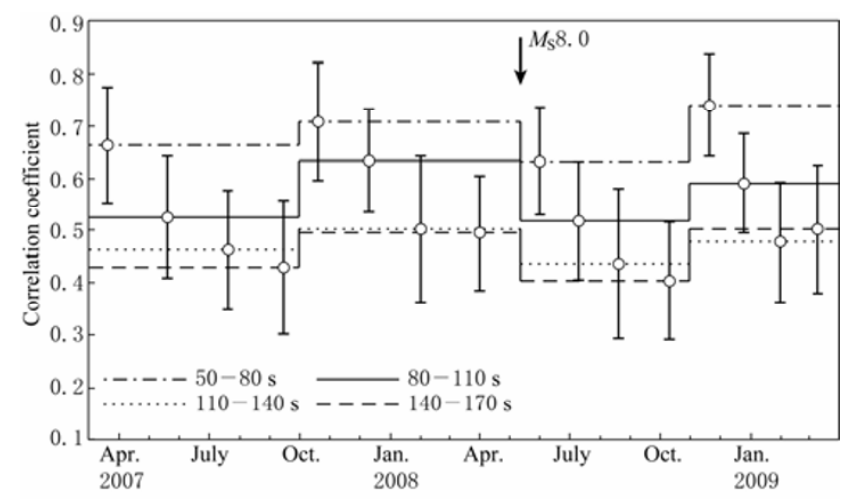

Figure 5 Correlation coefficient of current and reference EGFs in different lapse time windows for 11-day stacks. The straight lines indicate the means of correlation coefficient at the corresponding time period and the error bars indicate their standard deviations. The different line styles denote the non-overlapping windows in lapse time.

Another key parameter is the length of the time window in which the local time shift or velocity change is calculated with three moving-window techniques. To achieve meaningful correlation or cross spectra, the length of window is usually several times of the domain wavelength (e.g., Poupinet et al., 1984; Meier et al.,
2010). But the length is not rigorous because of the various spectra in different windows. We tested different window lengths, such as $30 \mathrm{~s}, 35 \mathrm{~s}$ and $40 \mathrm{~s}$, and have found the results very similar to each other. In this paper, we choose $30 \mathrm{~s}$ as the length of the time window.

The clock error or instrumental time shift is needed to be considered in using PII. The travel time shift caused by physical change in the medium would be symmetrical about zero point of EGF, whereas the clock error would result in a larger travel time in the positive and a smaller travel time in the negative time or vice versa (Stehly et al., 2007). It is noteworthy that the clock error herein is the asymmetric offset of travel time with respect to reference EGF, thus the error may not only result from the error in GPS receiver's clock, but also relate to the instrumental time shift or the seasonal migration of sources of the microseism (Stehly et al., 2007). Although Brenguier et al. (2008b) and Liu and Huang (2010) carried out the clock error corrections, the influences of clock error on measuring velocity change were not reported. We computed the clock errors by averaging the travel time shifts in the positive and negative parts of EGF in the lapse time range of 50-110 s (Figure 6). Two obvious time shifts, about $0.1 \mathrm{~s}$, both occur at the same time periods of the decrease of correlation coefficient: one is after the main shock; the other is in the summer of 2007. This is an interesting result which means the clock error can result in the decrease of correlation coefficient. However, whether the estimate of velocity change is affected by the clock error is still a question. We will compare the results with and without clock er-
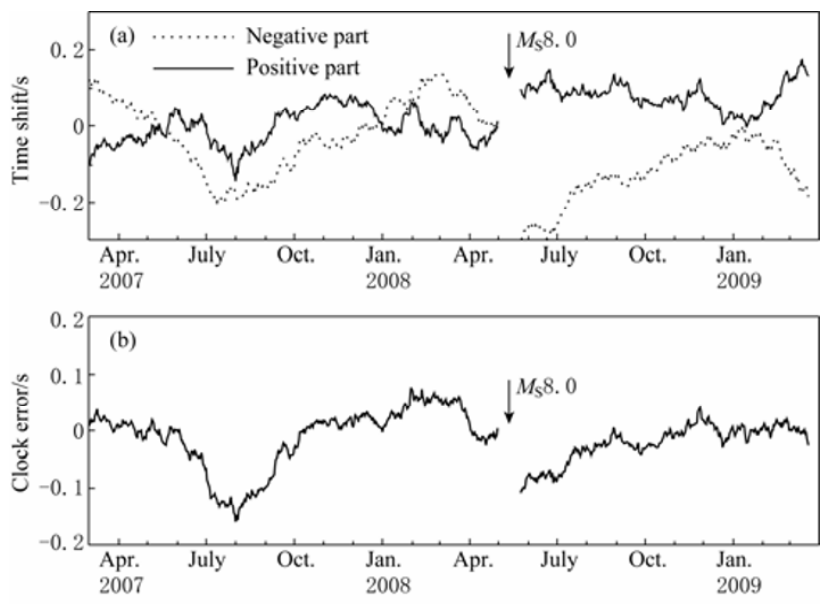

Figure 6 Clock error of current EGF for 61-day stacks. (a) Travel time shifts in negative and positive parts of EGF, respectively. (b) Temporal variations of clock error obtained by averaging the time shifts in the positive and negative parts of EGF. 
ror correction taking the 61-day stacks for example. Table 1 gives a summary of the computational parameters for estimating the temporal variations of seismic velocity in this paper.

Table 1 The parameters used for estimating temporal variation of seismic velocity in this paper

\begin{tabular}{|c|c|c|c|c|c|}
\hline $\begin{array}{l}\text { Stack time of } \\
\text { current EGF }\end{array}$ & Technique type & Range of lapse time/s & Length of window/s & Step of moving-window/s & Clock error correction \\
\hline 11-day & $\begin{array}{l}\text { MW } \\
\text { ST }\end{array}$ & $\begin{array}{c}{[-110,-50],[50,110]} \\
{[50,110]}\end{array}$ & $\begin{array}{l}30 \\
60\end{array}$ & $\begin{array}{l}1 \\
-\end{array}$ & $\begin{array}{l}\text { Yes } \\
\text { Yes }\end{array}$ \\
\hline 31-day & $\begin{array}{l}\text { MW } \\
\text { ST }\end{array}$ & $\begin{array}{c}{[-110,-50],[50,110]} \\
{[50,110]}\end{array}$ & $\begin{array}{l}30 \\
60\end{array}$ & $\begin{array}{l}1 \\
-\end{array}$ & $\begin{array}{l}\text { Yes } \\
\text { Yes }\end{array}$ \\
\hline 61-day & $\begin{array}{l}\text { MW } \\
\text { ST }\end{array}$ & $\begin{array}{c}{[-110,-50],[50,110]} \\
{[50,110]}\end{array}$ & $\begin{array}{l}30 \\
60\end{array}$ & $\begin{array}{l}1 \\
-\end{array}$ & $\begin{array}{l}\text { Yes/No } \\
\text { Yes/No }\end{array}$ \\
\hline
\end{tabular}

Note: MW contains MWCCT, MWCST and MWST. For ST, the casual and acasual parts of EGF are averaged.

\subsection{Results comparison}

Figure 7 shows the temporal variations of relative seismic velocity for station pair AXI-JJS in the 0.1 to $0.5 \mathrm{~Hz}$ frequency range. The result includes the measurements from 11-day, 31-day and 61-day stacks, each of which is computed with four techniques: MWCCT, MWCST, ST and MWST, respectively. We can detect the co-seismic sudden drop of velocity and the post-seismic recovery of velocity associated with the $M_{\mathrm{S}} 8.0$ Wenchuan earthquake using these techniques. But there are still differences among the results from the different techniques.

As for 11-day stacks (Figure 7a), the measurements with all techniques are scattered day by day because of the weak coherent waveforms used. MWCST provides a relatively clear trend of post-seismic velocity, whereas MWCCT provides a poor estimate. It seems that the signal of co-seismic velocity change is obscured in the long-term variation when ST is adopted.

As for 31-day stacks (Figure 7b), the sudden drop of seismic velocity at the time immediately after the Wenchuan earthquake is detected by using all techniques. And the best estimate is from MWCST due to the smallest error. There are some small but similar changes of seismic velocity, such as the increasing velocity in January 2008, obtained with all techniques, which are worth further analyzing.

When the stack time is up to 61-day (Figure 7c), the difference of velocity change is tiny for the three moving-window techniques. The post-seismic recovery trend of seismic velocity is well delineated, but some details of short-term velocity change disappear. For ST, there is obvious long-term signal in relative seismic velocity.

Figure $7 \mathrm{~d}$ shows the estimate of velocity change for 61-day stacks without clock error correction. In comparison with Figure $7 \mathrm{c}$, there is little difference for
MWCCT and MWST. In the result from MWCST, a small velocity drop appears in July 2007, which fades after clock error correction. Furthermore, the correction has a major impact on estimating the velocity change with ST. Without clock error correction, the sudden drop of seismic velocity associated with the Wenchuan main shock is observed by using ST, but stronger long-term variations appear compared to the result with clock error correction.

\section{Discussion and conclusions}

We compare the performance of four techniques (MWCCT, MWCST, ST and MWST) for estimating the velocity change with PII, using the data from a typical station pair near the Wenchuan fault zone. Our result is of practical significance to detect the velocity change in the microseism frequency band with PII.

The techniques based on moving-window are superior in detecting the change of seismic velocity because repeating process in multiple windows improves the stability of measurements and provides a quantitative estimate of the error of velocity change; whereas for single-window ST, the quality of measurements is only evaluated by the maximum of cross-correlation. In addition, it seems that the long-term variations are brought into.

Among the three moving-window techniques, MWCST can give a stable result of velocity change and small computing error under the circumstances of relatively low SNR and correlation coefficient of EGF (Figures $7 \mathrm{a}, 7 \mathrm{~b})$. However, for the data with higher quality (Figure 7c), the similar results are obtained with the three techniques, which suggests that the choice of techniques, by calculating the relative time shifts or stretching coefficient, will have not significant effect on the measurements of velocity change in this case. 

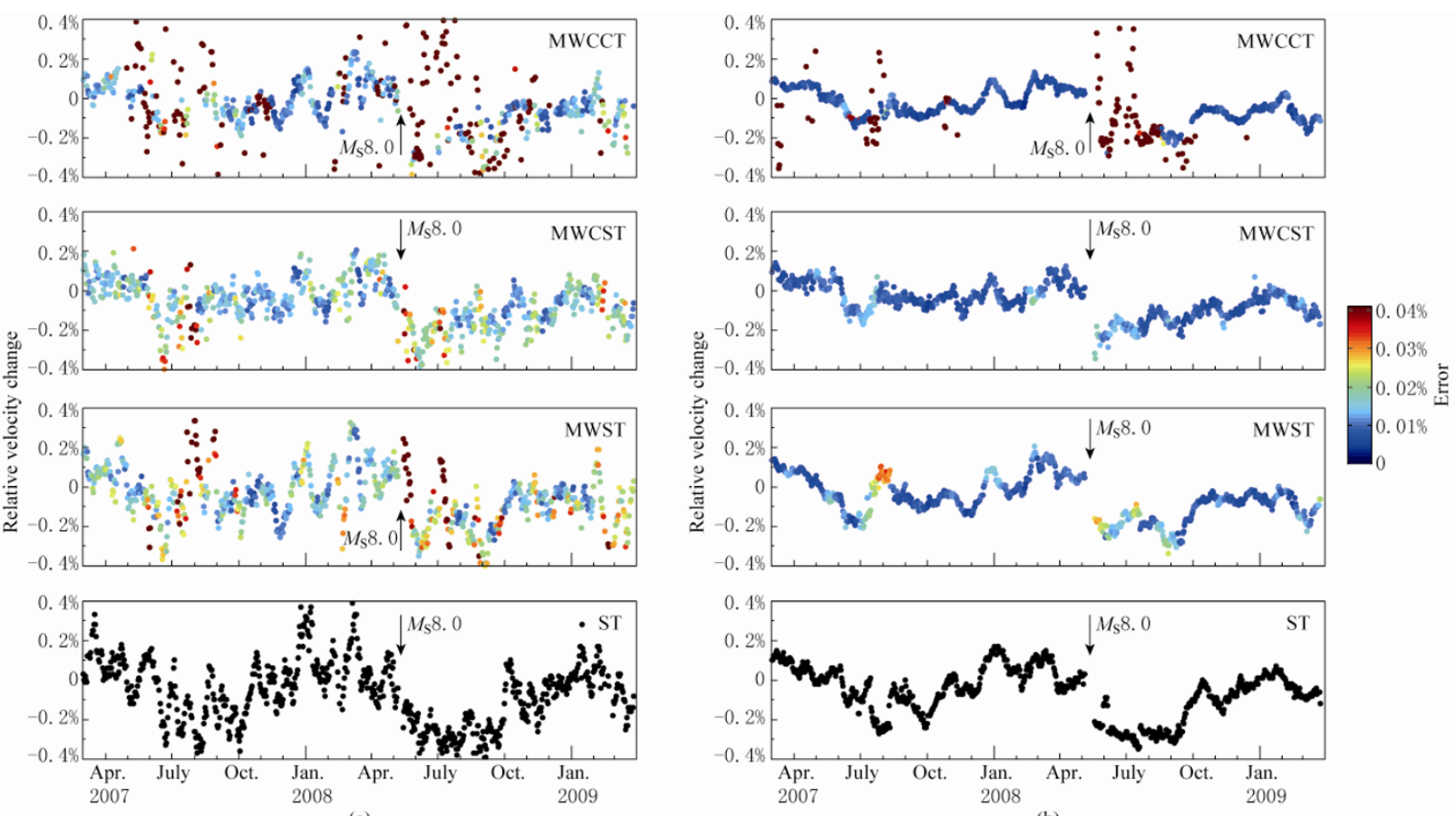

(b)
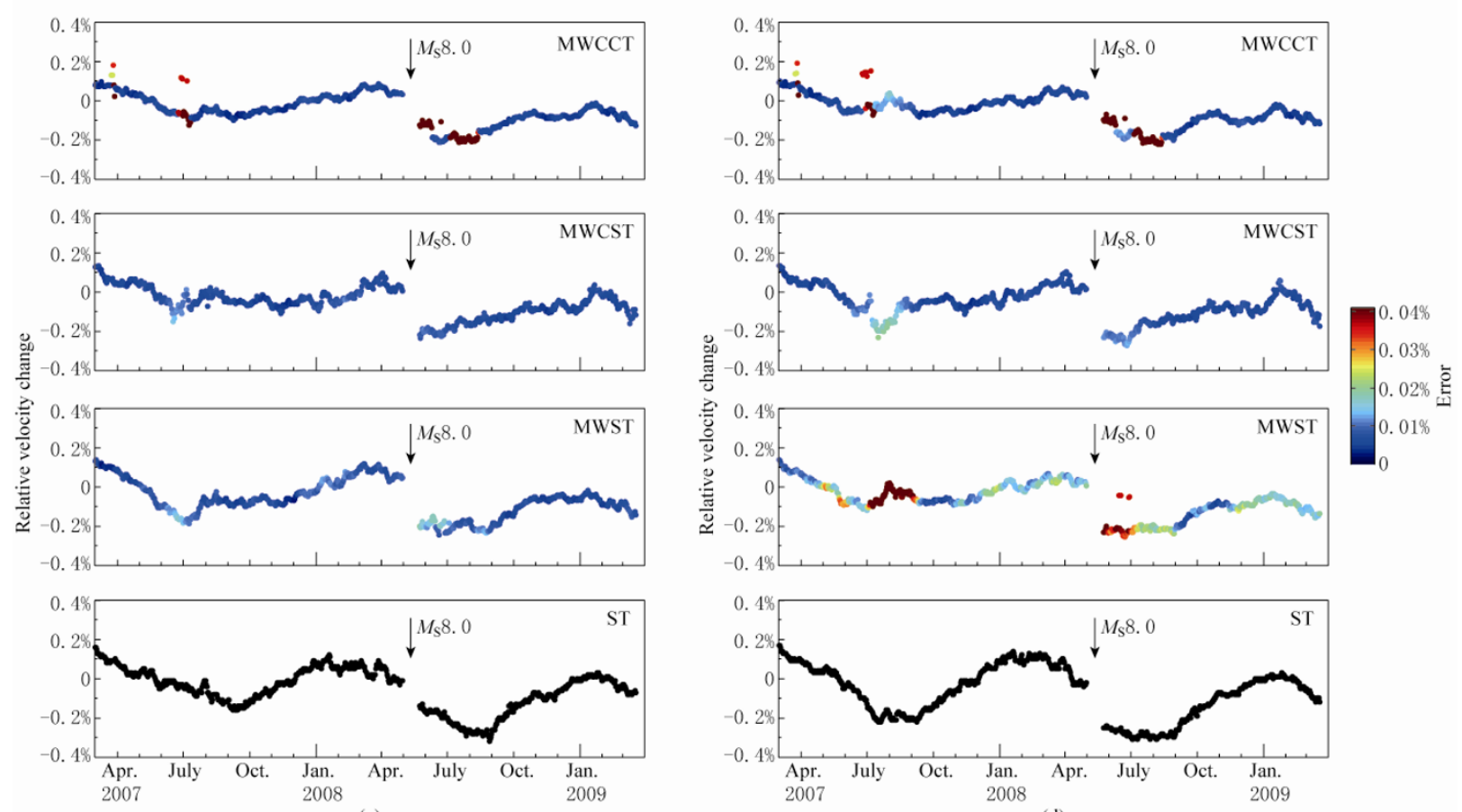

(d)

Figure 7 Relative seismic velocity changes with different techniques. (a)-(c) represent the relative seismic velocity changes for stacks of 11-day, 31-day and 61-day, respectively. (d) Same as (c), but without clock error correction. The name of technique is shown at the top right corner of each panel. The colors of points indicate the errors of measurements for the moving-window techniques and black points are used for ST because of no quantitative error estimate.

The clock error and its influence on velocity change are investigated in this paper. The clock error could result in the decrease of correlation coefficient of current EGF. When the clock errors are not very large (about $0.1 \mathrm{~s}$ ), they may have impact on estimate of velocity change using single-window ST, but have limited impact on the estimate of the velocity change in the frequency $0.1-0.5 \mathrm{~Hz}$ with moving-window techniques. 
However, the clock error correction is recommended for all computation techniques with PII because the correction can improve the results of velocity change to some extent and the correction value itself is an indicator of the quality of results.

Acknowledgements We are grateful to the editor Xiaodong Song and two anonymous reviewers for their constructive comments to improve the manuscript. The waveform data are provided by China Earthquake Network Center and Earthquake Administration of Sichuan Province. This research is supported by National Natural Science Foundation of China (No. 41074061) and Basic Research Plan of the Institute of Earthquake Science, China Earthquake Administration (No. 2007-13).

\section{References}

Aggarwal Y P, Sykes L R, Armbruster J and Sbar M L (1973). Premonitory changes in seismic velocities and prediction of Earthquakes. Nature 241: 101-104.

Bensen G D, Ritzwoller M H, Barmin M P, Levshin A L, Lin F, Moschetti M P, Shapiro N M and Yang Y (2007). Processing seismic ambient noise data to obtain reliable broad-band surface wave dispersion measurements. Geophys J Int 169(3): $1239-1260$.

Brenguier F, Shapiro N M, Campillo M, Ferrazzini V E R, Duputel Z, Coutant O and Nercessian A (2008a). Towards forecasting volcanic eruptions using seismic noise. Nature Geoscience 1: 126-130.

Brenguier F, Campillo M, Hadziioannou C, Shapiro N M, Nadeau R M and Larose E (2008b). Postseismic relaxation along the San Andreas fault at Parkfield from continuous seismological observations. Science 321: 1 478-1 481.

Duputel Z, Ferrazzini V, Brenguier F, Shapiro N, Campillo M and Nercessian A (2009). Real time monitoring of relative velocity changes using ambient seismic noise at the Piton de la Fournaise volcano (La Réunion) from January 2006 to June 2007. J Volcanol Geoth Res 184: 164-173.

Grêt A, Snieder R, Aster R C and Kyle P R (2005). Monitoring rapid temporal change in a volcano with coda wave interferometry. Geophys Res Lett 32: L06304.

Hadziioannou C, Larose E, Coutant O, Roux P and Campillo M (2009). Stability of monitoring weak changes in multiply scattering media with ambient noise correlation: Laboratory experiments. J Acoust Soc Am 125(6): 3 688-3 695.

Liu Z K and Huang J L (2010). Temporal changes of seismic velocity around the Wenchuan earthquake fault zone from ambient seismic noise correlation. Chinese J Geophys 53(4): 853-863 (in Chinese with English abstract).

Meier U, Shapiro N M and Brenguier F (2010). Detecting seasonal variations in seismic velocities within Los Angeles basin from correlations of ambient seismic noise. Geophys J Int 181: 985-996.

Peng Z and Ben-Zion Y (2006). Temporal changes of shallow seismic velocity around the Karadere-Düzce branch of the North Anatolian fault and strong ground motion. Pure Appl Geophys 163: 567-600.

Poupinet G, Ellsworth W L and Frechet J (1984). Monitoring velocity variations in the crust using earthquake doublets: An application to the Calaveras fault, California. $J$ Geophys Res 89(B7): 5 719-5 731.

Ratdomopurbo A and Poupinet G (1995). Monitoring a temporal change of seismic velocity in a volcano: Application to the 1992 Eruption of Mt. Merapi (Indonesia). Geophys Res Lett 22(7): 775-778.

Reasenberg P and Aki K (1974). A precise, continuous measurement of seismic velocity for monitoring in situ stress. $J$ Geophys Res 79(2): 399-406.

Sabra K G, Gerstoft P, Roux P, Kuperman W A and Fehler M C (2005). Extracting time-domain Green's function estimates from ambient seismic noise. Geophys Res Lett 32: L03310.

Schaff D P and Beroza G C (2004). Coseismic and postseismic velocity changes measured by repeating earthquakes. $J$ Geophys Res 109: B10302.

Semenov A N (1969). Variation in the travel time of transverse and longitudinal waves before violent earthquakes. Bull Acad Sci USSR Phys Solid Earth 3: 245-248.

Sens-Schönfelder C and Wegler U (2006). Passive image interferometry and seasonal variations of seismic velocities at Merapi Volcano, Indonesia. Geophys Res Lett 33: L21302.

Shapiro N M and Campillo M (2004). Emergence of broadband Rayleigh waves from correlations of the ambient seismic noise. Geophys Res Lett 31: L7614.

Snieder R (2006). The theory of coda wave interferometry. Pure Appl Geophys 163: 455-473.

Snieder R, Grêt A, Douma H and Scales J (2002). Coda wave interferometry for estimating nonlinear behavior in seismic velocity. Science 295: 2 253-2 255.

Stehly L, Campillo M and Shapiro N M (2006). A study of the seismic noise from its long-range correlation properties. $J$ Geophys Res 111: B10306.

Vidale J E and Li Y G (2003). Damage to the shallow Landers fault from the nearby Hector Mine earthquake. Nature 421: 524-526.

Wang B, Zhu P, Chen Y, Niu F and Wang B (2008). Continuous subsurface velocity measurement with coda wave interferometry. J Geophys Res 113: B12313.

Wegler U and Sens-Schönfelder C (2007). Fault zone monitoring with passive image interferometry. Geophys $J$ Int 168(23): $1029-1033$.

Wegler U, Nakahara H, Schönfelder C S, Korn M and Shiomi K (2009). Sudden drop of seismic velocity after the $2004 M_{\mathrm{W}} 6.6$ Mid-Niigata earthquake, Japan, observed with passive image interferometry. J Geophys Res 114: B06305.

Whitcomb J H, Garmany J D and Anderson D L (1973). Earthquake prediction variation of seismic velocities before the San Francisco earthquake. Science 180: 632-635.

$\mathrm{Xu} \mathrm{Z}$ and Song X (2009). Temporal change of surface wave velocity associated with major Sumatra earthquakes from ambient noise correlation. Proc Nat Acad Sci 106(34): 14 20714212. 